EXEMPLARIa Classica

Journal of Classical Philology

17, 2013, pp. 71-92

ISSN 1699-3225

\title{
IL MS. LEIDENSIS VOSSIANUS LATINUS F. 25 NELLA TRADIZIONE MANOSCRITTA DI VIRGILIO*
}

\author{
GIUSEPPE RAMIRES \\ Messina \\ g.ramires@tiscali.it
}

\section{SUMMARY}

The ms. Leidensis Voss. F. 25, written in France in the late ninth or early tenth century, contains the works of Virgil and the commentary by Servius. The manuscript, which belonged to Alexandre Petau and to Queen Christina of Sweden, may have come from the Monastery of Fleury. The present paper includes an examination of its relationship with the manuscript tradition of Virgil, both ancient and medieval. Study of the most interesting variants.

KEYWORDS

Virgil's text, manuscript tradition, critical edition.

\section{RESUMEN}

Il ms. Leidensis Voss. F. 25, scritto in Francia alla fine del IX o all'inizio del X secolo, contiene le opere di Virgilio e il commento di Servio. Appartenuto ad Alexandre Petau e in seguito alla Regina Cristina di Svezia, esso proviene forse dal Monastero di Fleury. Esame dei suoi rapporti con la tradizione manoscritta virgiliana più antica e medievale. Studio delle varianti più interessanti.

Palabras Clave

Testo di Virgilio, tradizione manoscritta, edizione critica.

Fecha de recepción: 04/07/2013

Fecha de aceptación y versión final: 01/09/2013

Dopo gli studi di Ribbeck, è invalso l'uso tra gli editori di Virgilio di affiancare, nella costituzione del testo, ai testimoni giunti dalla tarda antichità $(\mathrm{MPRAFGV})^{1}$, un certo numero di manoscritti di età carolingia. Mynors ne

* Sono grato agli amici Giancarlo Abbamonte, Luis Rivero García e Fabio Stok, che hanno letto questo contributo in abbozzo e mi hanno fornito preziosi suggerimenti, e Silvia Ottaviano, recentissima editrice delle Bucoliche, che mi ha messo a disposizione tutte le collazioni pubblicate nel database "Manuscripta Vergiliana, I codici di Virgilio tra IX e XI secolo": www. sns.it/ricerca/lettere/manoscrittivirgilio/.

${ }^{1} \mathrm{M}=$ Mediceus Laurentianus lat. Plut. XXXIX, $1+$ Vaticanus lat. $3225-\mathrm{II}$ (fol. lxxvi = Aen. 8.585-642), scritto a Roma nel 494-495; $\mathrm{P}=$ Vaticanus Palatinus lat. 1631, scritto in Italia alla fine del V o all'inizio del VI sec.; $\mathrm{R}$ = Vaticanus lat. 3867 , chiamato "Romanus", scritto 
impiegò tredici ${ }^{2}$, Geymonat ne aggiunse uno nella sua prima edizione ${ }^{3}$, e altri tre nella seconda del 20084. Dopo di lui, Conte è arrivato (per la sola Eneide) a quota ventitré ${ }^{5}$, mentre l'Ed. della Coll. Alma Mater si è fermata a diciotto ${ }^{6}$. La recentissima ed. di Bucoliche e Georgiche, curata rispettivamente da S. Ottaviano e G. B. Conte ${ }^{7}$, ha introdotto un nuovo interessante tassello: al cod. n, scritto probabilmente a Napoli nella seconda metà del X sec. ${ }^{8}$, i due stu-

a Roma o a Ravenna nel VI sec.; A = Vaticanus lat. 3256 + Berolinensis lat. Fol. 416, scritto forse a Roma nel VI sec.; F = Vaticanus lat. 3225 "Fulvii Ursini schedae Vaticanae", scritto a Roma alla fine del IV sec.; $\mathrm{G}=$ Sangallensis 1394, scritto forse in Italia all'inizio del VI sec.; $\mathrm{V}=$ Veronensis XL 38, scritto forse in Italia settentrionale nel $\mathrm{V}$ sec. A questi mss. tutti più o meno incompleti, va aggiunto $\mathrm{B}=$ Palimps. Ambrosianus L 120 sup., del V sec. Precedenti l'età carolingia, scritti sul finire dell'VIII sec., rimangono anche $\mathrm{m}=$ Monacensis lat. 29216/7, scritto in Italia, e p = Parisinus lat. 7906, scritto in Germania occidentale. Dall'ed. di Conte (cf. infra) adottiamo i simboli per indicare le diverse mani correttrici, soprattutto in $\mathrm{M}$ e $\mathrm{P}$.

${ }^{2}$ P. Vergili Maronis Opera, recognovit brevique adnotatione critica instruxit R. A. B. Mynors, Oxonii 1969: $\mathrm{a}=$ Bernensis $172+$ Parisinus lat. 7929 , metà sec. IX; $\mathrm{b}=$ Bernensis 165 , sec. IX ${ }^{1 / 4} ; \mathrm{c}=$ Bernensis 184, sec. X; d = Bernensis 255 e $239+$ Parisinus lat. 8093 , sec. $\mathrm{IX}^{2 / 303 / 3} ; \mathrm{e}=$ Bernensis 167, sec. IX $\mathrm{IX}^{2} \mathrm{f}=$ Oxoniensis Bodl . auct. F. 2. 8., sec. IX ${ }^{1} ; \mathrm{h}=$ Valentia nensis 407 (389), sec. IX ${ }^{2} ; \mathrm{r}=$ Parisinus lat. 7926, sec. IX ${ }^{2 / 4} ; \mathrm{s}=$ Parisinus lat. 7928, sec. IX o IX/X; $\mathrm{t}=$ Parisinus lat. 13043, sec. IX ${ }^{2 / 3} ; \mathrm{u}=$ Parisinus lat. 13044, sec. IX ex.; v = Vaticanus lat. 1570, sec. IX/X. A questa lista va aggiunto il cod. $\gamma=$ Guelferbytanus Gudianus lat. $2^{\circ}$ 70, sec. IX, apografo del Palatinus.

${ }^{3}$ P. Vergili Maronis Opera, post Remigium Sabbadini et Aloisium Castiglioni recensuit M. Geymonat, Torino 1973: $\mathrm{n}=$ Neapolitanus Vind. lat. 6, sec. X.

${ }^{4}$ P. Vergili Maronis Opera, edita anno MCMLXXIII iterum recensuit M. Geymonat, Roma 2008: k = Hamburgensis Scrin. 52, metà del IX sec.; o = Ausonensis, sec. XI ${ }^{1}$ (è il più antico testimone spagnolo di Virgilio, le cui varianti non sono qui registrate; il siglum o è utilizzato per un ms. di Oxford, cf. infra); x = Montepessulanus Fac. Med. 253. I codd. k x furono da Geymonat aggiunti alla lista ma non collazionati. Per o lo studioso si serviì invece delle colazioni di M. Librán Moreno, "Colación del ms. 197 («P. Vergilii Maronis Bucolica Georgicon Aeneidos») del Archivo Capitular de Vic”, ExClass 9, 2005, 22-73.

${ }^{5}$ P. Vergilius Maro, Aeneis, recensuit G. B. Conte, Berolini et Novi Eboraci 2009: sono stati aggiunti oltre a k x (collazionati), anche $\mathrm{g}=$ Parisinus lat. 7925 , sec. IX ${ }^{2 / 4} ; \mathrm{i}=$ Reginensis 1669, sec. IX metà; $\mathrm{j}=$ Bruxellensis Bibl. reg. 5325-5327, sec. IX ${ }^{2 / 3} ; \mathrm{w}=$ Guelferbytanus Gudianus 66, sec. IX ${ }^{1} ; y=$ Parisinus lat. 10307, sec. IX ${ }^{4 / 4} ; z=$ Parisinus lat. 7927, sec. X. Conte impiega anche i frammenti del codice $\mathrm{q}=$ Berolinensis lat $.2^{\circ} 421+$ Monacensis lat $.29216 / 8$ + fragmentum Monacense ex libris Bernardi Stark 18, VIII.

${ }^{6}$ Publio Virgilio Marón, Eneida, introducción, texto latino, traducción y notas de L. Rivero García-J. A. Estévez Sola- M. Librán Moreno- A. Ramírez de Verger, vol. I (libros I-III), Madrid 2009; vol. II (libros IV-VI), Madrid 2011; vol. III (libros VII-IX), Madrid 2011; vol. IV (libros X-XII), Madrid 2011: a b br (= j) c d e f h k n r s t u v x g o.

${ }^{7}$ P. Vergilius Maro, Bucolica, ed. S. Ottaviano - Georgica, ed. G. B. Conte, Berlin-Boston 2013. Per le Bucoliche, la Ottaviano impiega gli antiquiores M P R V, e i carolingi già usati da Conte per l'Eneide, ad eccezione di q u perché non hanno le Bucoliche. La studiosa aggiunge $1=$ Vaticanus lat. 3252 , sec. XIV ex e due frammenti siglati $\varphi_{1}=$ Valentianensis 178 (170), sec. IX ${ }^{1}$. Per le Georgiche, Conte impiega gli antiquiores M P R A F G V e i codici carolingi come la Ottaviano (più j che ha invece le Georgiche), ad eccezione ovviamente del frammento $\varphi_{1}$.

${ }^{8}$ Si tratta del Neapolitanus Lat. 6, realizzato a Napoli - come il Neapolitanus Lat. 5 e, forse, il Parisinus Lat. 10308 - in scrittura beneventano-cassinese, per la biblioteca del Duca di Napoli, Giovanni III (928-968/9): questi manoscritti sarebbero, secondo il paleografo Gugliel- 
diosi accostano nell'apparato critico altri sei manoscritti vergati in scrittura beneventana, tra il X e l'XI secolo9. Il consensus di tali mss. è indicato con $\Lambda$, quello dei codd. carolingi diventa invece $\Phi$. Il siglum $\omega$ viene conservato per indicare l'accordo di $\Lambda \Phi$. Per il presente lavoro, verrano utilizzati come testo-base l'ed. di Conte per l'Eneide e quella di Ottaviano e Conte per Bucoliche e Georgiche.

L'impiego dei manoscritti di epoca carolingia e di area beneventanocassinese è generalmente motivato in diversi modi: anzitutto, alcuni di essi sono considerati apografi più o meno diretti di codici tardoantichi conservati e possono pertanto supplirli quando questi ultimi presentano lacune ${ }^{10}$; in un certo numero di casi - non moltissimi, in verità - sembrano conservare la lezione corretta contro i guasti della tradizione più antica11; in altri casi vengono a sostegno di lezioni ottime tramandate da un solo testimone tardoantico o dalla tradizione indiretta ${ }^{12}$; in ultimo, costituiscono di per sé un punto fermo nella storia della tradizione del testo virgiliano ${ }^{13}$, cosa non trascurabile, vista la grande incidenza che l'opera di Virgilio ha avuto sulla cultura medievale. Alcuni decenni fa, uno studioso statunitense ha tentato di stabilire una 'familiarità' tra undici manoscritti carolingi ${ }^{14}$ : pur circoscritta all'Eneide, l'operazione ha dato qualche esito positivo, ma la non infondata identificazione di tre gruppi non può tuttavia prescindere da una vastissima contaminazione, che impedisce di stabilire, per ogni singolo gruppo, un capostipite 'sicuro'15.

Dopo l'affondo di Conte, e di Ottaviano e Conte, sono pochi i mss. del IX-X secolo rimasti fuori dalla recensio ${ }^{16}$ : fra questi figura il codice della $\mathrm{Bi}$ -

mo Cavallo, la testimonianza di una tradizione napoletana di studi su Virgilio, che forse non si era del tutto estinta nei secoli V-IX e che, comunque, era stata rinvigorita durante il ducato. Cf. G. Cavallo, "La trasmissione dei testi nell'area beneventano-cassinese", in La cultura nell'Occidente latino dal VII all'XI secolo, Spoleto, 357-414: 380-1; G. Abbamonte, "Diligentissimi vocabulorum perscrutatores". Lessicografia ed esegesi dei testi classici nell'Umanesimo romano di XV secolo, Pisa 2012, 148.

${ }^{9} \mathrm{v}=$ Neapolitanus Vindob. Lat. 5 , sec. $\mathrm{X}^{1}\left(\mathrm{o} \mathrm{IX}^{\mathrm{ex}}\right) ; \mathrm{o}=$ Oxoniensis Canon. Class. 50, sec. $\mathrm{XI}^{3 / 4} ; \delta=$ Parisinus lat. 10308 , sec. $\mathrm{XI}^{3 / 4} ; \varepsilon=$ Vaticanus lat. 1573 , sec. XI; $\zeta=$ Vaticanus lat. 3253, sec. XI $; \eta=$ Reginensis lat. 2090 , sec. $\mathrm{XI}^{\mathrm{ex}}$.

${ }^{10}$ Oltre a $\gamma$, apografo di $\mathrm{P}$, anche a apografo di R.

${ }^{11}$ Per un elenco - limitato all'Eneide - di quelle accolte nell'ed. di Conte (con riferimenti alle edizioni di Mynors, Geymonat e a quella della Coll. Alma Mater), cf. la mia recensione in Vergilius 58, 2012, 124-5. Rimane il dubbio se queste 'buone lezioni' siano frutto di congetture o derivino da testimoni tardoantichi perduti.

${ }^{12}$ Cf. G. Ramires, in Vergilius 58, 2012, 125-6, sempre limitatamente all'Eneide.

${ }^{13}$ Nell'area continentale per $\Phi$, in Italia per $\Lambda$.

${ }^{14}$ R. A. Kaster, The Tradition of the Text of the Aeneid in the Ninth Century, New York-London 1990: b r (gruppo I), d f h o t (gruppo II, o = j), a e u v (gruppo III).

${ }^{15}$ Cf. L. D. Reynolds, "Virgil", in L. D. Reynolds, ed., Texts and Transmission. A Survey of the Latin Classics, Oxford 1983, 433-6.

${ }^{16} \mathrm{E}$ persiste, naturalmente, il dubbio, che qualche ms. dell'XI o del XII o del XIII secolo - per non spingersi sino ai codici umanistici - possa anch'esso tramandare 'buone lezioni', 
bliotheek der Rijksuniversiteit di Leiden, Vossianus Latinus F. 25, scritto in Francia - e databile alla fine del IX o all'inizio del X secolo ${ }^{17}$. Tale manoscritto di formato 328 x $255 \mathrm{~mm}$., si compone di 178 fogli e contiene, dal f. $4^{\mathrm{r}}$ al f. $175^{\mathrm{r}}$ le Bucoliche (f. $4^{\mathrm{r}}-15^{\mathrm{r}}$ ), le Georgiche (f. $15^{\mathrm{r}}-43^{\mathrm{v}}$ ) e l'Eneide (f. $44^{\mathrm{v}}-175^{\mathrm{r}}$ ) scritte su una colonna di testo al centro del foglio (per lo più di 38 versi), con il Commento di Servio redatto tutt'intorno sui margini superiore e inferiore, sinistro e destro ${ }^{18}$. Il ms. Leidensis apparteneva alla raccolta di Isaac Voss nei f. $2^{\mathrm{r}}$ e $3^{\mathrm{r}}$ si legge un'etichetta cartacea a stampa, incollata, "Ex bibliotheca Viri Illustris Isaaci Vossii", con un'aggiunta a mano del n. "39" -, o meglio a quella parte della collezione, messa insieme da Voss per la regina Cristina di Svezia, che non raggiunse Roma - e la Biblioteca Vaticana - ma nel 1690 finì, com'è noto, a Leiden ${ }^{19}$. Voss formò la grande collezione acquistando tra gli altri i libri del giurista francese Paul Petau dal figlio di questi Alexandre nel $1650^{20}$. Come afferma De Meyier, il Leidensis Voss. F. 25 proveniva proprio dalla raccolta di Alexandre Petau ${ }^{21}$. Si potrebbe trattare dunque di uno dei manoscritti direttamente acquistati da quest'ultimo, ma rimane il dubbio e la possibilità che si tratti invece di uno dei manoscritti della biblioteca di Paul Petau, il quale aveva a sua volta comprato una parte dei libri del giureconsulto di Orléans Pierre Daniel ${ }^{22}$. Questi aveva fatto il colpo della sua vita quando, nel 1562, dopo il saccheggio da parte degli Ugonotti, era riuscito ad acquistare un certo numero di codici dell'Abbazia di Fleury nel nord della Francia. Sappiamo che tra i manoscritti di Fleury appartenuti a Daniel vi era l'attuale Bernensis 172 + Parisinus 7929 (noto come Floriacensis), che contiene il testo di Virgilio (sigla a) e in margine il commento di Servio ampliato ad Aen. 3-12 (sigla F, sec. IX). Ora, il Leidensis Voss. F. 25 è un manoscritto che, come

intelligenti congetture.

${ }^{17}$ Cosi K. A. De Meyier, Codices Vossiani Latini, I, Codices in folio, Leiden 1973, 52-6. $\mathrm{Al}$ sec. X lo assegna, invece, B. Munk Olsen, L'étude des auteurs classiques latins aux XI et XII ${ }^{e}$ siècles, Catalogue des manuscrits classiques latins copiés du IXe au XII e siècle, Paris 1985, II, 727. A questi due lavori si rinvia per una descrizione completa del manoscritto.

${ }^{18} \mathrm{Il}$ Commento di Servio alle Bucoliche va dal f. $3^{\mathrm{v}}$ a $14^{\mathrm{v}}$; quello alle Georgiche da $15^{\mathrm{r}}$ a $42^{\mathrm{v}}$; quello all'Eneide da $44^{\mathrm{v}}$ a $173^{\mathrm{r}}$. I ff. $176^{\mathrm{r}}-9^{\mathrm{v}}$ contengono il commento di Servio ad Aen. 5.7206.107, che colma una lacuna prodottasi nel f. 91v.

${ }^{19}$ L. D. Reynolds-N. G. Wilson, Copisti e filologi. La tradizione dei classici dall'antichità ai tempi moderni, trad. di M. Ferrari, Padova 1973, 191. Per gli avvenimenti che portarono i manoscritti di Voss a Leiden, cf. De Meyier, Codices, X.

${ }^{20}$ Cf. K. A. De Meyier, Paul en Alexandre Petau en de geschiedenis van hun handschriften, Leiden 1947.

${ }^{21} \mathrm{Il}$ n. 162 che si legge «in tergo tegumenti» suggerisce a De Meyier, Codices, 55, un rinvio al f. $83^{v}$ del Catalogo manoscritto, conservato a Leiden come Voss. Lat. Q. 76, «in quo Alexander ipse non paucas adnotationes de codicibus postea acquisitis addiderat», De Meyier, Codices, XI. Al manoscritto è peraltro premesso un foglio cartaceo, del sec. XVI, che contiene un indice scritto dallo stesso Alexandre Petau, cf. De Meyier, Codices, 53.

${ }^{22}$ Reynolds-Wilson, Copisti, 182. 
ho avuto altre volte occasione di riferire ${ }^{23}$, contiene circa quattrocento aggiunte che prima erano note soltanto grazie a $\mathrm{F}$ (e ad alcuni altri testimoni meno completi). Queste aggiunte le ho rinvenute anche nel Parisinus 7961 (Pc) e, per una piccola sezione, in un frammento conservato come foglio di guardia del Reginensis $1495\left(\mathrm{r}^{24}\right)$. I due mss. formano la classe che ho denominato $\alpha$ : essa - conservando come ho già detto circa quattrocento aggiunte prima note solo grazie al Floriacensis - ha con quest'ultimo una importante relazione. Non è questo il luogo per sviluppare un ragionamento sulla possibilità che il Leidensis o il suo antigrafo abbia contribuito alla formazione del Floriacensis - cosa possibile ma difficilmente dimostrabile (e naturalmente ci sarà chi penserà che le cose stiano esattamente al contrario) -, ma si può ipotizzare che il Leidensis provenga anch'esso da Fleury e sia passato dalle mani di Daniel25

${ }^{23}$ Cf. G. Ramires, "Per una nuova edizione di Servio", RFIC 124, 1996, 318-29; G. Ramires, ed., Servio, Commento al libro IX dell'Eneide di Virgilio, Bologna 1996, XXXI-XXXII; G. Ramires, ed., Servio, Commento al libro VII dell'Eneide di Virgilio, Bologna 2003, XXXVI-XLIX, LXVII.

${ }^{24}$ Il frammento potrebbe essere ciò che resta di un manoscritto di Virgilio + Servio, scritto in Germania (Munk Olsen, L'étude, 780) o in Francia (E. Pellegrin, Les manuscrits classiques latins de la Bibliothèque Vaticane, Paris 1975-1982, 1.2, 240-2) tra l'XI e il XII secolo. Dato che è attualmente foglio di guardia del Reginensis 1495, si può ipotizzare che il frammento (o addirittura l'intero manoscritto originale) abbia avuto lo stesso passaggio di mani che portò molti manoscritti alla Biblioteca Apostolica Vaticana attraverso Daniel, Petau, Vossius e la Regina Cristina (il ms. Reg. 1495 apparteneva sicuramente al fondo della Regina Cristina, cf. Pellegrin, Les manuscrits, 242 = n. 1660 del catalogo Montfaucon), e che pertanto vada posto in una più stretta relazione con il Leidensis Voss. F. 25. In effetti nel Catalogo di Montfaucon dei manoscritti della Regina (Bibliotheca Bibliothecarum manuscriptorum nova, 1, Paris 1739 = Les manuscrits de la Reine de Suède au Vatican. Réédition du catalogue de Montfaucon et cotes actuelles, Città del Vaticano 1964) c'è l'indicazione di un codice che conteneva Virgilio e Servio e del quale non c"è più traccia. Cf. G. Ramires, "Per una nuova edizione di Servio", RFIC 124, 1996, 318-29: 320-1; G. Ramires, ed., Servio, Commento al libro IX dell'Eneide di Virgilio. Con le aggiunte del cosiddetto Servio Danielino, Bologna 1996, XXXII.

${ }^{25}$ Non è possibile dimostrare in assoluto che Daniel abbia posseduto ed eventualmente impiegato il Leidensis Voss. F. 25, tuttavia si possono addurre alcuni indizi di carattere testuale. Premesso che le aggiunte di $\alpha$ furono in gran parte pubblicate da Robert Estienne (Stephanus) nel 1532 (68 anni prima dell'ed. di Daniel, cf. G. Ramires, "Il «Servius Danielinus» prima di Pierre Daniel: l'edizione di Robert Estienne (Stephanus) e i manoscritti della classe $\alpha$ ", Eruditio Antiqua 4, 2012, 137-203: www.eruditio-antiqua.mom.fr) e che il testo tramandato da F risulta in più punti malandato, si può cercare di approfondire la natura degli interventi emendatori di Daniel. In molti casi la correzione può essere derivata a Daniel proprio dall'ed. dello Stephanus, per es.: Aen. 3.151.13 Harv. circa Le Pc Steph. Dan. : ciria F; 4.144.10<ut> Le Pc Steph. Dan. : om. F; 4.531.2 et est sententia Le Pc Pa Steph. Dan. : hsentia et est F G; 5.758 .11 consultum Le Pc Pa W Steph. Dan. : evanuit in F; 5.840.3-4 in undas constat missum Le Pc Pa ${ }^{2}$ Steph. Dan. : in undas cecidisse constat missum F G C; 7.188.8 Ram. acus Le Pc Steph. Dan. : aius F; 7.188.9 veientorum Le $\mathrm{Pc} \mathrm{Pa}^{2}$ Steph. Dan. : venientorum F. Ci sono poi dei casi in cui il testo o l'emendazione pubblicati da Daniel trovano riscontro soltanto in Le (e nel suo gemello Pc): 8.409 (261. 20 Th.) subtili Le Dan. : subtilio F sub stilio G; 8.666 (196.24 Th.) uti non Le Pc $\mathrm{Pa}^{2}$ Dan. : utin F. Naturalmente, in molti se non in tutti questi casi - e in altri che qui si tralasciano - si può anche avanzare l'ipotesi di un'autonoma attività emendatoria di Daniel. Ciò però risulterebbe più difficile a 10.220 (416.1 Th.) rotatione Le $\mathrm{Pc} \mathrm{Pa}$ Dan. : ratione F G (una mano 
in quelle di Petau, prima di Paul e poi di Alexandre e da questi a Voss, approdando infine alla Bibliotheek der Rijksuniversiteit di Leiden. Il Leidensis fu compulsato da Nicolaus Heinsius per il testo di Servio e le sue lezioni furono riportate, come afferma Burman, con la sigla $\mathrm{V}$ e $\mathrm{L}^{26}$. La scrittura è una bella carolina, un po' allungata, di modulo leggermente inferiore per il commento di Servio. Sia il testo di Virgilio che quello di Servio sono stati vergati da più mani $^{27}$. Le lettere iniziali dei libri virgiliani sono in una elegante semionciale, che viene imitata anche - ma non sempre - per i lemmi di Virgilio nel commento di Servio. La mia collazione, eseguita su un microfilm in mio possesso, rivela alcune significative peculiarità del Leidensis: il rilevante grado di contaminazione - peculiare di tutti i manufatti di quell'età - non ne consente una sicura collocazione in uno dei tre gruppi secondo la semplificazione di Kaster; d'altra parte il Leidensis si distingue per un certo numero di lezioni singolari - alcune a quanto sembra del tutto sconosciute, altre attestate come congetture - e per altre già confermate da uno o più mss. tardoantichi e da uno (o due) mss. carolingi. Si preferisce iniziare dalle lezioni singolari, adottando per il Leidensis il siglum Le, che già lo caratterizza nelle mie edizioni di Servio.

\section{Bucoliche}

1.18 sed tamen iste deus qui sit, da, Tityre, nobis qui : quis Le

Geymonat segnala quis in apparato come congettura di Earle ${ }^{28}$, ma questa variante è accolta, ad es., da de La Cerda, e «ha ampio riscontro negli incunaboli» ${ }^{29}$.

2.46 ecce ferunt Nymphae calathis; tibi candida Nais

tibi: et Le

\subsection{0 instituit, Daphnis thiasos et ducere Bacchi}

Bacchi : Bacho (sic) Le

Geymonat ricorda che Baccho è lez. di alcune edizioni a stampa ${ }^{30}$, ma

recente corregge in F) e c'è almeno un caso in cui la correzione o l'intera aggiunta pubblicata da Daniel non si riscontra in F ma soltanto in Le Pc e nell'ed. dello Stephanus: 7.790 .9 faciebat: sua pictura insignem (insigne Steph.) faciebat add. Le Pc Steph. Dan. C’è però anche un caso, piuttosto significativo, in cui Daniel pubblica il testo corrotto, mentre Le Pc Pa hanno la lezione corretta: 12.41 (580.12 Th.) remotio Le Pc Pa : remo F Dan.

${ }^{26}$ Cf. De Meyier, Codices, 55.

${ }^{27}$ Cf. De Meyier, Codices, 52-53.

${ }^{28}$ M. L. Earle, "Observatiunculae ad locos quosdam poetarum Romanorum”, $R P h$ n.s. 27, 1903, 269-72.

${ }^{29}$ Già nell'ed. di Strasburgo del 1470 (n. 3 di M. Davies-J. Goldfinch, Vergil. A Census of Printed Editions 1469-1500, London 1992, 39), da cui dipende l'ed. di Colonia del 1470 (n. 102 Davies-Goldfinch, p. 79), cf. M. Venier, Per una storia del testo di Virgilio nella prima età del libro a stampa (1469-1519), Udine 2001, 139.

${ }^{30}$ Questa variante si trova già nell'editio princeps (Roma. 1469, = n. 1 Davies-Goldfinch, 
conclude nescio qua auctoritate. Ł̀ evidente che il genitivo va benissimo - Dafni stabilì di introdurre le processioni "di Bacco" - ma persisterebbe l'ombra di un pleonasmo. Di chi altri sarebbero i thiasi se non di Bacco? ${ }^{31}$ Per questo motivo anche a me non sembra del tutto peregrino quel Baccho, come dativo di vantaggio, nel senso di "a Bacco", "in onore di Bacco"32. Cfr. Aen. 7.580-581 tum quorum attonitae Baccho nemora avia matres / insultant thiasis, dove però sembra ablativo ${ }^{33}$.

Georgiche

1.20 et teneram ab radice ferens, Silvane, cupressum

$a b: a$ Le

Ma $a b$ radice si legge in Georg. 2.17 e Aen.12.787, cfr. anche $a b$ radicibus di Georg. 1.319.

1.339 sacra refer Cereri laetis operatus in herbis

herbis : arvis Le

Cfr. Aen. 6.744 pauci laeta arva tenemus ${ }^{34}$.

2.285 non animum modo uti pascat prospectus inanem inanem : inane Le

2.353 hoc, ubi hiulca siti findit Canis aestifer arva hoc : atque Le

3.331 aestibus at mediis umbrosam exquirere vallem at $\mathrm{F}^{2} \mathrm{M} \omega:$ ad $\mathrm{R}$ a t $\mathrm{v} \gamma$ ac $\mathrm{f} \mathrm{gh} \mathrm{j}$ aut $\mathrm{F} \mathrm{P} \mathrm{b} \mathrm{r} \varepsilon$ in $\zeta$ Non. 247.11 et Le

3.449 et spumas miscent argenti vivaque sulpura

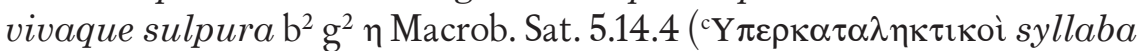
longiores sunt $)^{35}$ Victorinus 212.21 Sch. Bern. Serv. viva et sulpura $\mathrm{r}$ sulphura vivaque $\mathrm{i}^{\mathrm{pc}}$ Le ordinem mutavit et sulp(h)ura viva M P R $\omega \gamma$ ac sulphura viva $\mathrm{n} \zeta$

39), "e di qui si propaga fino almeno alle prime tre edizioni Aldine", Venier, Per una storia, 139.

${ }^{31}$ Servio spiega infatti saltationes, choreas Liberi, id est Liberalia.

${ }^{32}$ Bacho leggo anche in o, dopo correzione (forse ex Bachi).

${ }^{33}$ Ottaviano preferisce invece intervenire su inducere che dopo instituit è considerato supervacaneum. La studiosa propone a testo l'emendazione et ducere (cf. anche S. Ottaviano, "Due nuove proposte per il testo delle Bucoliche (ecl.3, 102; 5, 30)", MD 67, 2011, 203-14).

${ }^{34}$ Erren conosce la variante arvis, che in apparato attribuisce a non meglio precisati «dett.». Cf. ad loc. M. Erren, ed., P. Vergilius Maro, Georgica, band I, Einleitung, Praefatio, Text und Übersetzung, Heidelberg 1985.

${ }^{35}$ Oltre a Georg. 3.449, Macrobio cita anche Aen. 6.33, Georg. 1.295 e 2.69. 
Il testo della trad. ms. più antica - et sulphura viva - attesta il tentativo di sanare un'anomalia metrica: dactylicus versus scrive infatti Servio e quod in fine dactylum habeat spiega l'aggiunta di $\mathrm{V}^{36}$.

\section{Eneide}

1.48 bella gero. Et quisquam numen Iunonis adorat

adorat $\mathrm{M} \gamma \mathrm{R} \omega \mathrm{Tib}$. Gramm. adorant $\mathrm{p}:$ adoret $\varepsilon \zeta \mathrm{Le}$

Negli apparati di Mynors, Geymonat, Conte, Coll. Alma Mater, la variante adoret era nota soltanto per trad. indiretta: Quint. 9.2.10, Serv. Georg. 4.502, DServ. Aen. 2.79, 10.826, 12.11; essa è invece conservata da Le e da due testimoni in beneventana, ed è accolta, per esempio, da Paratore (Milano 1978), che conserva il successivo futuro imponet ${ }^{37}$.

\subsection{6 fervet opus redolentque thymo fraglantia mella}

fraglantia $\mathrm{P}$ a b e g h j k v w $\gamma$ n o $\delta \eta$ : flagrantia F M R c d f i r t x y $\mathrm{z} \varepsilon \zeta$ $v$ codd. Diomedis 319.18 fragrantia $\mathrm{M}^{\mathrm{P}}$ Le Serv. DServ. codd. Macrob. sat. 5.11.2 Prisc. 8.95, 9.43 Isid. orig. 17.9.1238.

\subsection{8 litora iactetur odiis Iunonis acerbae}

iactetur odiis a $\mathrm{z} \zeta$ iactaetur odiis $\mathrm{F}$ : iacteturque odiis B $\mathrm{F}^{2} \mathrm{M} \gamma \mathrm{R} \Pi_{5}$ $\omega$ (periit in $\mathrm{P}$ ) Non. 328.5 Serv. Tib. iacteturque dolis Le

Quasi tutti i mss. hanno iacteturque (iactetur a z $\zeta$ iactaetur F, ma lo stesso Servio scrive vacat 'que' e l'allungamento in arsi di -tur è adottato da Virgilio anche in Georg. 3.76, Aen. 4.222, 5.284 39); dolis di Le - pur metricamente corretto - ha l'aria di essere un errore d'origine paleografica (metatesi, lettere soprascritte?), con un certa plausibilità riguardo al senso: Enea è "sbattuto in giro sui mari da una costa all'altra" ${ }^{40}$ per l'odio ma anche per gli

\footnotetext{
${ }^{36} \mathrm{~V}$ legge in finem, ma la correzione in fine, ancor prima che da Fulvio Orsini (Roma 1587) è introdotta nell'ed. serviana del 1471, probabilmente da Guarino Veronese, dove si legge anche $q u i$ al posto di quod. Per Guarino Veronese editore di Servio, cf. G. Ramires, "Guarino Veronese editore di Servio e il problema delle cosiddette 'aggiunte danieline", in C. Santini-F. Stok, edd., Esegesi dimenticate di autori classici, Pisa 2008, 113-33; G. Ramires, "News of Guarino's Lost Servius", in S. Casali-F. Stok, edd., Servius: Exegetical Stratifications and Cultural Models, Bruxelles 2008, 224-48.

${ }^{37}$ Nell.'ed. di Paratore (Milano 1978, Della Corte traduce "Qualcuno venererebbe ancora il nume di Giunone, o supplice porrebbe offerte sulle are?". Adoret sembra invece suggerire a De La Cerda di accogliere la correzione imponat dei codd. Recentiores. D'altra parte, con una lunga, problematica e interessante nota, Conington-Nettleship (1884, p. 10) sostengono la loro preferenza per adorat / imponit, lezione quest'ultima di p, di quasi tutti i codd. del nono-decimo secolo e (forse) di Tiberio Donato.

${ }^{38}$ Per una discussione sul valore della variante fragrantia, che fu posta a testo - dopo Ribbeck e Sabbadini - ancora da Goold (Harvard 1999), cf. G. Ramires, recensione dell'ed. di Conte e della Coll. Alma Mater, 130.

${ }^{39}$ Cf. R. S. Conway, ed., P. Vergili Maronis Aeneidos liber primus, Cambridge 1935, 114.

${ }^{40}$ Così traduce R. Scarcia (Milano 2002, 319).
} 
inganni e le insidie di Giunone. Nel suo discorso al figlio Cupido, la dea Venere dice dolis subito dopo al v. 673, e ciò può aver procurato l'errore a 668 . Qualcuno potrebbe pensare ad un addensamento voluto - ci sono anche dolos e dolo al v. 682 e al v. 684 -, ma è difficile che sia così. Per l'uso di dolus cfr. anche Aen. 1.130, 4.95, 128, 8.393.

\subsection{1 et lateri adglomerant nostro, iuvenisque Coroebus}

et : se Le

Enea si lancia tra le armi e si uniscono - addunt se - Rifeo e Ifito; e poi Hypanisque Dymasque et lateri adglomerant nostro. Sicuramente adglomerant vale per se adglomerant, da qui la congettura di Peerlkamp confermata ora da Le - cfr. Aen. 12.457-58 -; certo, l'ellissi del pronome non è inusuale in Virgilio, cfr. Aen. 2.229, 235, 11.707¹, tuttavia c'è una certa durezza in quell'et.

\subsection{6 forsitan et Priami fuerint quae fata requiras} requiras : requires Le

La variante è attestata da Charis. 239.16 B., ma cfr. Georg. 2.288 forsitan ... quaeras.

\subsection{8 fundit humi, duo lacte novo, duo sanguine sacro} fundit : fudit Le

La lezione fuolit è conservata anche da Arusiano 42.6 Di Stefano ${ }^{42}$.

\subsection{2 unde locum Grai dixerunt nomine Aornum}

Aornum k $\gamma^{43}$ : Avernum $\mathrm{R}$ a b x o $\delta^{2} \zeta^{2} \eta \vee$ Aornon ed. Aldina an. 1501 Averna Le

Il verso 6.242 è tramandato, tra gli antiquiores, dal solo $\mathrm{R}$, e da pochi testimoni medievali, a b k o x $\gamma$ (a $\gamma$ prima del v. 241), cui si aggiunge adesso Le;

${ }^{41}$ Cf. F. Speranza, ed., Eneide II, Napoli 1964, 112.

42 "Solo l'operetta di Arusiano trasmette il perfetto fudit" scrive A. Di Stefano, ed., Arusiani Messi Exempla Elocutionum, Introduzione, testo critico e note, Hildesheim 2011, 134. Il testo di Arusiano è il seguente: FUNDIT HUMI. Virg. Aen. V 'Mero libans carchesia Baccho fudit humi'. Nel lemma fundit è lez. sicura, mentre su fudit nella citazione gli editori precedenti erano divisi: fudit pubblicò Keil nei GL (seguito da Marmorale), fundit preferì invece Della Casa, Milano 1977. La Di Stefano, credo giustamente, pubblica fudit e precisa in apparato che fundit legge il solo $\mathrm{V}^{1}$ (= Vaticanus Lat. 3402, sec. XVI in.), correggendo così l'ed. Della Casa, che invece attribuiva fundit anche a $\mathrm{V}^{2}$ (=Vaticanus Lat. 5216, sec. XVI), A (= Ambrosianus D 498 inf., sec. XVI - datato al sec. XVIII da Di Stefano nel Conspectus siglorum di p. 3, per un mero errore tipografico; corretta invece la datazione, sec. XVI, nella descrizione del ms. a p. LXXIV), P (= Leidensis Perizonianus Q. 84, sec. XVIII).

${ }^{43} \mathrm{La}$ lez. Aornum è attestata anche nelle aggiunte alla Vita serviana che si trovano nel Vaticanus lat. 3252 della fine del IX sec., cf. J. J. H. Savage, "The Manuscripts of the Commentary of Servius Danielis on Virgil”, HSCPh 43,1932,115-6, e più recentemente Venier, Per una storia, 13-16. 
è stato inserito, probabilmente da Pomponio Leto, nel Mediceo - $\mathrm{M}^{\mathrm{P}}$ - e da un correttore d'età umanistica in $\mathrm{F}-\mathrm{F}^{5}$. Studiosi ed editori sostanzialmente concordano nel ritenerlo spurio e ne propongono pertanto l'atetesi. Per Averna sostantivo cfr. Aen. 3.442, 5.732 e 7.91, ma anche Lucr. 6.738, Ov. met. 14.105, DServ. Aen. 5.732.

\subsection{3 admoneat volitare cava sub imagine formae}

cava: cavae Le

La variante di Le fu congetturata da Bentley ${ }^{44}$ (il cod. $\eta$ legge cava, ma $\eta^{2}$ supra lineam scrive vel ve ovvero vel cave $)^{45}$. La Sibilla spiega a Enea che i mostri che lui vede sono vite senza corpo che fluttuano cava sub imagine formae. Nonostante il confronto con Aen. 1.516 (nube cava) e 2.360 (cava umbra), intendere letteralmente "sotto la vuota immagine di una forma" è meno efficace di "sotto l'immagine di una vuota forma". In effetti cava va benissimo, inteso con ipallage, cioè con riferimento logico a formae. La correzione - pensata da Bentley e adesso attestata da Le - risulta probabilmente pleonastica, ma viene ancora citata nell'apparato di Geymonat ${ }^{46}$.

\subsection{8 haec erat illa fames, haec nos suprema manebat}

manebat : canebat Le

Quando Ascanio pronuncia la famosa frase - etiam mensas consumimus - Enea capisce di aver raggiunto la tanto agognata mèta, si ricorda della profezia - dell'arpia Celeno non di Anchise - e aggiunge haec erat illa fames, haec nos suprema manebat, exitiis positura modum. Con canebat la frase acquisterebbe un altro significato: bisognerebbe fare una pausa più decisa dopo fames e sottintendere Anchise come soggetto. Non tanto male, insomma, ma il nesso suprema (sost o agg.) canere non sembra attestato in latino classico. La variante può essere sorta anche per evitare una cacofonia suprema manebat. Per canebat in clausola - nove occorrenze in Virgilio -, cfr. in particolare Aen. 3.559 haec saxa horrenda canebat, profezia di Eleno.

\subsection{0 iamque iter emensi turris ac tecta Latinorum}

\section{Latinorum : Latinum a b e x n $v^{\mathrm{pc}}$ Latini $\delta^{\mathrm{pc}}$ Le}

I cento ambasciatori inviati da Enea scorgono le torri e gli ardua tecta dei Latini; ma la variante Latini - quindi, del re Latino - è perfettamente congrua, la si legge nel Mediceo, quale probabile correzione di Pomponio Leto

${ }^{44}$ A. Stachelscheid, "Bentley's Vergiliana”, 312-3.

${ }^{45}$ Cavae si legge già nell'ed. Romana del 1471 (n. 4 Davies-Goldfinch, p. 40), cf. Venier, Per una storia, 56 e 140.

${ }^{46}$ Dalla nota di Burman ad loc. (Amsterdam 1746, 52-3) si evince che la variante cavae dovrebbe trovarsi anche in altri due mss.: "CAVA. Cavae formae prior Vossianus et Rottendorphius primus. HEINS. Cavae etiam Leidensis unus». 
- $\mathrm{M}^{\mathrm{P}}$ - e piacque ad alcuni editori, giustamente e meritoriamente segnalati nell'apparato critico della Coll. Alma Mater ${ }^{47}$.

7.232 fama levis tantique abolescet gratia facti tantique F M P b f g $\gamma$ $\eta$ : tantive $\mathrm{R} \omega \mathrm{Tib}$. tanti aut Le

Cfr. Aen. 1.566 virtutesque virosque aut tanti incendia belli?

\subsection{0 te liquidi flevere lacus}

lacus : et Megarus ipse add. Le; supra lineam mons est Le

Questo completamento di uno dei cinquantotto tibicines dell'Eneide non sembra altrimenti noto ${ }^{48}$. Megarus fa difficoltà per il metro (bisognerebbe accettare un allungamento in arsi), un po' meno per il senso, se ipotizziamo una sua origine basso medievale. La parola, infatti, indicherebbe l'isolotto di fronte a Napoli ${ }^{49}$, ora Castel dell'Ovo, un luogo simbolo di Napoli, denso di leggendari richiami virgiliani ${ }^{50}$.

9.620 Idaeae: sinite arma viris et cedite ferro

ferro : bello Le

\subsection{1 aspice num mage sit nostrum penetrabile telum} num : nunc Le Diom. 441.33 Serv.

${ }^{47}$ Gli editori sono O. Güthling (Lipsiae 1894), P. Deuticke apud Th. Ladewig (Berlin 1904, più precisamente C. Schaper: «Sch. las nach der spaeten Korrektur in M Latini», p. 276), G. P. Goold (Cambridge 2000).

${ }^{48}$ Per questo verso si conosceva, invece, il completamento nemorosaque Tempe. Per un'analisi di questo tibicen, con utili confronti, cf. A. Novara, "Alcune osservazioni sui versi incompiuti nell'Eneide di Virgilio: cenni sul lavoro di Virgilio tragico”, Aevum 67, 1993, 37-53, in part. 49. Dei completamenti spurii, Le riporta soltanto 5.595 luduntque per undas $(=\mathrm{R}$ c e $\mathrm{x}$ y $\gamma^{1}$ ). Sui tibicines dell'Eneide, cf. la relativa voce in $E V$, vol. $V^{*}$, Roma 1990, 167-70, curata da V. Viparelli, con altra bibliografia.

${ }^{49}$ L'antico nome Megaris (Plin. nat. 3.82 Inter Pausilypum et Neapolim Megaris) o Megalia (Stat. Silv. 2.2.80 quaeque ferit curvos exerta Megalia fluctus), deriva dal tufo di cui si compone l'isolotto, ma Pausania 1.40.1 racconta che il giovane Megarus, figlio di Giove e di una ninfa, per sfuggire il diluvio di Deucalione, segui delle gru che si fermarono proprio sulla cima di quel monte, che prese il nome di Gerania (dal nome greco delle gru) o di Megarus. In verità, per il contesto virgiliano (la morte del marso Umbrone), ci si aspetterebbe che una qualche montagna, e non un isolotto, si unisse al pianto del bosco di Angizia, del Fucino e dei limpidi laghi. Forse per questo motivo l'amanuense di Le si affretta ad aggiungere nell'interlineo mons est. La discrasia potrebbe suggerire l'origine dell'integrazione in un ambito influenzato dalle leggende sul "mago" Virgilio.

${ }^{50}$ Cf. J. M. Ziolkowski-M. C. J. Putnam, The Virgilian Tradition. The first fifteen hundred years, New Haven-London 2008, 932-3, dove si riassume la digressione virgiliana di Castel dell'Ovo narrata nel Roman de Cleomadés dal poeta e menestrello francese J. Adenet Le Roi (circa 1240-circa 1300), e passim. 


\subsection{9 invidisse deos, patriis ut redditus aris}

aris : atris Le arvis $\delta \zeta$ agris $\varepsilon^{51}$

All'assemblea di guerra presieduta da Latino, Venulo fa rapporto della sua ambasceria presso Diomede; vengono riferite le parole dell'eroe greco che, prima di rifiutare l'aiuto richiesto e di consigliare, al contrario, un accordo coi Troiani, ricorda a quali tragedie abbia condotto la guerra di Ilio e quali furono i drammatici ritorni degli eroi argivi; a lui stesso, l'ostilità divina proibì di tornare patriis aris e di rivedere così la desiderata sposa ${ }^{52}$ e la bella Calidone. Il termine ara è usato da Virgilio quasi sempre in senso proprio, col significato di altare; qui varrebbe come una sineddoche. La lezione introdotta da Le atris (con contrazione) potrebbe anche sembrare più allettante, ma il nesso patria atria non è attestato in latino classico. Gli atria sarebbero le stanze e dunque il palazzo del padre, cfr. nell'Eneide 2.483 (atria longa), 528 (vacua atria) - il palazzo di Priamo - descritto nel momento supremo della morte del grande re e del figlio Polite. Da notare che anche nella altre quattro occorrenze virgiliane, la parola atria è collegata a personaggi particolarmente drammatici: a Didone in 1.726 (ampla atria) e 4.666 (alta atria, per cui va la Fama) e in due similitudini, la prima, 7.379, per Amata (vacua atria), la seconda, 12.474, per Giuturna (alta atria) ${ }^{53}$. Anche nel racconto di Diomede prevalgono gli argomenti drammatici: il ricordo degli atria paterni si legherebbe a quello malinconico e penoso del tradimento della moglie e della patria perduta; ma rimane forte la difficoltà a cui prima si accennava.

11.671 suffosso revolutus equo dum colligit, alter

suffosso M b i r n $\delta \varepsilon v$ alii ap. Serv. ${ }^{54}$ : suffuso M R $\omega$ Serv. Tib. subfuso $\gamma$ suffusso Sabbadini Le

${ }^{51}$ Per patriis ... aris, nesso già enniano (scaen. 84 J., arae patriae), cf. Aen. 3.332 (patriasque obtruncat ad aras) e il commeno a 11.269 di N. Horsfall, ed., Virgil, Aeneid 11. A Commentary, Leiden-Boston 2003, 186.

${ }^{52}$ Virgilio dice coniugium optatum. Durante l'assenza di Diomede, impegnato nella guerra di Troia, la moglie Egialia aveva commesso adulterio con Cillarabo, figlio di Stenelo.

${ }^{53}$ Cf. M. Bettini, "Turno e la rondine nera", QUCC 30, 1988, 7-24. Prima ancora, per il confronto con i vacua atria di Polite (Aen. 2.528), cf. M. C. J. Putnam, The Poetry of the Aeneid, Cambridge Mass. 1965, 172.

${ }^{54}$ In realtà suffosso è lez. del solo Floriacensis $(\mathrm{F})$, gli altri codici serviani hanno suffuso. Ma siccome suffuso è già la lez. del lemma e qui Servio sembra voler introdurre una diversa lezione sostenuta da non meglio identificati alii, non è del tutto peregrino immaginare che l'archetipo serviano avesse proprio suffusso. Si potrebbe tuttavia formulare una diversa ipotesi, ponendo l'accento sulla differenza tra il testo di Servio e Servio auctus (DS): suffosso nel lemma di Servio (come in effetti si legge nei codd. $\alpha$ ) e suffuso nel lemma DS; alii 'suffuso' legunt in Servio, alii 'suffosso' legunt in DS. 
L'emendazione di Sabbadini è adesso confermata da Le. Conte dà preferenza a suffosso anche per il confronto con Caes. Gall. 4.12 .2 (subfossisque equis), Tac. ann.1.65.6 (suffosso equo), 2.11.3 (suffosso equo).

Accordo di Le con uno o più degli antiquiores contro il consenso della tradizione medievale:

Bucoliche

6.51 et saepe in levi quaesisset cornua fronte

quaesisset $\mathrm{M} \mathrm{R} \omega \gamma$ : quesissent $\mathrm{P} \varphi_{1}$ Le, quaesissent Sch. Bern. Ribbeck, Sabbadini

Georgiche

2.181 Palladia gaudent silva vivacis olivae

gaudent $\mathrm{P} \mathrm{b} \mathrm{k} \gamma^{2}$ Le : gaudet $\mathrm{M} \omega$ Ter. Maur. 1222

2.443 navigiis pinus, domibus cedrumque cupressosque

pinus $\mathrm{P} \mathrm{cd} \gamma \mathrm{Le}$ : pinos M R $\omega \gamma^{1}$

L'acc. pl. pinus è sicuramente usato da Virgilio a Aen. 9.116 e $11.136^{55} \mathrm{e}$ probabilmente a $B u c .8 .22^{56}$; è giusto accogliere pinus anche in questo passo, come fa Conte e come aveva del resto già fatto Mynors.

3.77 primus et ire viam et fluvios temptare minacis

minacis $\mathrm{R}$ minaces $\mathrm{M} \omega \gamma$ : minantis $\mathrm{P}$ minantes $\mathrm{Le}$

Conte, come già Mynors. prefersce fluvios temptare minacis, ma la scelta rimane difficile e minantis leggeva Seneca che cita Georg. 3.75-81 e 83-85 a ep. 95.68.

3.395 ipse manu salsasque ferat praesepibus herbas ipse $\mathrm{M} \gamma$ Le ipsese $\mathrm{V}$ : ille P R $\omega$

\section{Eneide}

5.564 nomen avi referens Priamus, tua clara, Polite

Polite $\mathrm{M}^{\mathrm{x}} \mathrm{P}^{\mathrm{x}} \mathrm{p} \omega \gamma$ Serv. Tib. Prisc. 7.6 : Polites M P R Le

Per l'uso del nominativo al posto del vocativo, in formule rituali, cfr. audi tu, populus Albanus in Liv. 1.24.7; in poesia cfr. almae filius Maia in Hor. od. 1.2.42-3; o populus in Luc. 2.116; $i$, pete virginea, populus in Ov. Fast. 4.731. In Virgilio, Aen. 8.77 corniger Hesperidum fluvius regnator aquarum, con il commento di Servio: vocativus antiquus est, qui apud

${ }^{55}$ Qui il solo M ha pinos.

${ }^{56}$ Mynors pubblica pinusque loquentis (così $\mathrm{P} \gamma$ ), mentre Geymonat preferisce pinosque loquentis seguendo M R $\omega$. C’è da considerare anche Georg. 4.112, dove gli editori pubblicano giustamente tinosque (M P), ma pinosque hanno invece $\mathrm{F} \mathrm{M}^{2} \omega \gamma$. 
maiores in omni forma similis erat nominativo. Cfr. anche Aen. 11.464 Messapus e Servio: ergo 'Messapus' aut antiquus vocativus est [...] aut certe nominativus est pro vocativo.

5.685 tum pius Aeneas umeris abscindere vestem abscindere P p $\omega \gamma$ Serv. Tib : excindere $\mathrm{M}$ abscidere $\mathrm{R}$ Le

7.161 ardua cernebant iuvenes muroque subibant

muroque : murosque R muro+que Le, an murosque ante corr.?

Murosque subibant ha il solo cod. R (anche $\zeta v^{\mathrm{ac}}$ ). Servio ammette che l'accusativo sarebbe più naturale, per dativum enim figuratum est. Gli editori sono soliti rinviare a Aen. 9.371 dove si trova la stessa clausola e però i mss. si dividono: muroque hanno M R g j n, murosque invece P V $\omega$ $\gamma$. Conte decide di uniformare (muroque) ${ }^{57}$ mentre Geymonat e l'Ed. Spagn. preferiscono murosque a Aen. 9.371.

10.683 fluctibus an iaciat mediis et litora nando iaciat : iaceat $\mathrm{d}$ i $\mathrm{\zeta}$ iactet $\mathrm{R}$ Le iacet $\mathrm{O}^{2}$

12.382 abstulit ense caput truncumque reliquit harenae harenae M P $\zeta$ Serv. Aen. 11.87 Tib. harene Le : harena R $\omega \gamma$

Accordo di Le con uno o più degli antiquiores e uno o due codici carolingi (o in beneventana):

Bucoliche

7.25 pastores, hedera nascentem ornate poetam

nascentem $\mathrm{V}$ b i s $\varepsilon$ Le Serv. nascente $\mathrm{M}$ crescentem $\mathrm{M}^{\mathrm{A}} \mathrm{P}$ a $\omega \gamma$ Ps. Acr. Hor. Carm. 1.1.29 Philarg. Serv. Buc. 4.19

Georgiche

1.296 et foliis undam trepidi despumat aeni

trepidi $\mathrm{P}^{2} \omega \gamma$ Gell., Ansil. AE 147, Sch. Bern. : trepidis M R tepidi $\mathrm{P}$ o $\eta \mathrm{Le}^{58}$.

Eneide

7.436 ore refert: classis invectas Thybridis undam

undam F M $\gamma \omega$ Tib. : unda $\mathrm{fg}$ h i j z undas $\zeta$ albeo $\mathrm{M}^{2}$ alveo $\gamma^{1} \mathrm{R} \mathrm{jac}$ o Le

La variante alveo può essersi prodotta - anche indipendentemente - per memoria della clausola di Aen. 7.303 Thybridis alveo.

8.75 fonte tenet, quocumque solo pulcherrimus exis

tenet M P $\omega \gamma$ Serv. : tenent $\mathrm{F} \mathrm{R}$ jac Le tenes $\mathrm{v}$ o Tib.

${ }^{57}$ Cf. C. E. Murgia, “An unrecognized Vergilian variation”, Hermes 116, 1988, 493-9.

${ }^{58}$ Lezione che godrà ampia fortuna nei manoscritti umanistici, cf. Venier, Per una storia, 6. 
8.90 ergo iter inceptum celerant rumore secundo celerant: peragunt $\mathrm{R}$ a j Le peragant $\gamma^{1}$ celebrant $\mathrm{g} \mathrm{n}$ o $\delta \eta \mathrm{v}^{\mathrm{pc}}$ Cfr. Aen. 6.384 ergo iter inceptum peragunt ${ }^{59}$.

10.696 ipsa immota manens, prolem Dolichaonis Hebrum manens : manent $\mathrm{M}$ manet $\mathrm{M}^{2}$ a $\mathrm{O}^{2} \varepsilon \mathrm{Le}$

11.23 mandemus, qui solus honos Acheronte sub imo est imo est $\mathrm{M}^{\mathrm{A}} \omega \gamma^{1}$ Serv. Tib. imost $\mathrm{M}$ : imo P R a Le

11.236 olli convenere fluuntque ad regia plenis fluuntque : ruuntque $\mathrm{M}$ b r v Le

11.381 quae tuto tibi magna volant, dum distinet hostem distinet $\mathrm{P} \omega \gamma$ Serv. Tib. : destinat $\mathrm{R}$ a j ${ }^{\mathrm{ac}}$ destinet $\varepsilon$ detinet $\mathrm{M} \times \mathrm{z} \zeta \vee \mathrm{Le}$

11.598 Etruscique duces equitumque exercitus omnis

Etruscique : Etrurique Pac d r v Le

La tradizione manoscritta attesta Etrusci (cfr. anche Aen. 9.150 e 10.429) e gli editori accolgono di solito questa lezione. Del resto, la variante Etruri (lectio difficilior?) sarebbe hapax, ma va detto anche che essa è attestata da Tiberio Donato nel lemma, ricordata favorevolmente da DServ.: quidam sane 'Etruri' legunt ${ }^{60}$, ab 'Etruria'; trans Tiberim enim Etruriam dicebant, homines Etruros, quos nunc Etruscos, e accolta da Sabbadini.

11.703 posse neque instantem reginam avertere cernit avertere : advertere $\mathrm{M}$ corr. $\mathrm{M}^{\mathrm{x}}$ vertere $\mathrm{R}$ a b Le

11.742 concitat et Venulo adversum se turbidus infert infert $\mathrm{M} \omega$ Serv. Aen. 11.814 : offert $\gamma \mathrm{R}$ a o Le Tib.

11.846 extrema iam in morte, neque hoc sine nomine letum neque : nec $\mathrm{R}$ a $\mathrm{n} \delta$ \& Le

12.470 excutit et longe lapsum temone reliquit reliquit $\mathrm{M}$ P R $\omega \gamma$ : relinquit $\mathrm{M}^{\mathrm{A}}$ ? $\mathrm{V}$ o $\zeta \mathrm{Le}$ 123.

${ }^{59}$ Cf. W. Moskalew, Formular Language and Poetic Design in the Aeneid, Leiden 1982, piunt.

${ }^{60}$ Forse ancor più incisivo il Turonensis $(\mathrm{T})$ : quidam 'Etruri' ab 'Etruria' dictum acci- 
Volendo trarre qualche conclusione sui dati fin qui proposti ed esaminati, si può dire che il ms. Le conserva molte lezioni tipiche della tradizione virgiliana carolingia (e in beneventana):

Buc. 1.72 his nos $\mathrm{P} \mathrm{R}$ a b $\gamma$ : en quis $\omega \gamma^{1}$ Le en quos $\mathrm{d} 1 ; 2.32$ primus $\Phi$ $\varepsilon \gamma$ Le : primum P R a $\Lambda ; 8.58$ fiat M P b f g r $\gamma^{1}$ : fiant a $\omega \gamma$ Le; 8.70 ulixi M P $1 \mathrm{rx}$ o: ulixis a $\omega \gamma$ Le;

Georg. 1.287 gelida melius M P R b j r s $\Lambda$ : melius gelida $\Phi$ Le; 1.341 tum M R b d f g r x $\Lambda$ : tunc $\Phi$ Le; 1.351 possemus M R b g j r : possimus $\mathrm{M}^{\mathrm{P}} \omega \gamma$ Le; 1.366 umbram $\mathrm{M} \gamma \mathrm{R}$ b j $\mathrm{r} \zeta$ : umbras $\omega$ Le; 1.383 variae $\mathrm{M} \gamma$ $\mathrm{R}$ a h 1 : varias $\omega \gamma^{1}$ Le; 2.52 voces $\gamma \omega$ Le : voles $\mathrm{M} \gamma^{1} ; 2.82$ miratastque $\mathrm{M}^{\mathrm{x}}$ : mirataeque $\mathrm{M}$ mirata estque a $\mathrm{d} \gamma^{2}$ mirataque $\gamma^{*}$ miratasque $\Phi$ Le miraturque $\mathrm{M}^{\mathrm{P}}$ e $\Lambda ; 2.200$ derunt $\mathrm{M} \mathrm{P}$ a b deerunt $\mathrm{M}^{\mathrm{x}} \mathrm{k}$ r x y desunt $\omega \gamma^{*}$ $\gamma^{2}$ Le; 2.219 semper viridi M P R b r n o $\eta$ : viridi semper $\omega \gamma^{*}$ Le; 2.365 nondum falcis M P R V b r $\Lambda$ : falcis nondum $\Phi \delta \zeta$ Le; 2.413 rusti $\mathrm{M}^{\mathrm{A}} \mathrm{P}$ $\mathrm{R} \mathrm{k} \mathrm{rtz?} \Lambda \gamma$ : etrusci M rusci $\Phi \varepsilon \gamma^{1}$ Le; 2.472 exiguoque M P R b r n $\zeta$ : parvoque $\omega \gamma$ Le; 3.73 statues M P R b r t o $\varepsilon \eta$ : statuis $\omega$ Le; 3.249 agris M P R b r $\Lambda$ : arvis $\Phi \delta$ Le; 3.288 hic labor F M P R b i r t $\Lambda$ : hinc labor $\Phi$ Le; 3.297 felicumque F M P R f g j $\mathrm{n}$ : filicumque $\omega \gamma$ Le; 3.396 et magis M P R V b v $\Lambda$ : ac magis $\Phi \delta \eta$ Le om. r; Georg. 4.20 inumbret M P R b r t $\zeta \gamma:$ obumbret $\omega \gamma^{1}$ Le adumbret $\mathrm{f} \mathrm{g} \mathrm{h} \mathrm{i} \mathrm{j} \delta$; 4.97 sicco terram F M P b r $\Lambda$ : terram sicco $\Phi \delta \varepsilon$ Le terram et sicco t; 4.135 etiamnum $\mathrm{M} \mathrm{r} \varepsilon \eta$ etiannum $\mathrm{P}$ : etiam nunc $\omega \gamma^{1}$ Le; 4.373 effluit M P R b $\varepsilon \eta$ (evan. in G) : influit $\omega \mathrm{Le} ; 4.431$ dispergit $\mathrm{M} \mathrm{R}$ : discerpsit $\mathrm{P}$ dispersit (des- $\mathrm{d} \mathrm{w}) \omega \gamma$ Le; 4.435 auditisque M P R b g h r: auditique $\omega \gamma$ Le;

Aen. 1.701 manibus famuli G P R c h j r $\gamma^{1} \mathrm{n}$ o $\varepsilon \zeta \eta$ : manibus famulae M P $\mathrm{P}^{1}$ famuli manibus $\mathrm{B} \omega$ Le; 2.30 acie M P R n $\gamma$ : acies $\Pi_{5} \omega \gamma^{1}$ Le; 2.661 isti M P V h j k v z n $\delta$ v: ista a? istic $\omega$ Le istinc $\eta ; 3.435$ proque $\mathrm{M} \mathrm{P} \mathrm{d} \mathrm{h} \mathrm{k}$ $\mathrm{n}$ o r t $\mathrm{w}$ y : praeque $\omega \gamma^{1} \mathrm{Le} ; 3.543$ et M P r t v x y $\gamma \zeta$ : ea c est $\omega$ Le; 3.558 haec $\Phi \gamma^{1}$ Le : hic M P d r tw $\gamma \Lambda$; 4.116 confieri $\gamma \mathrm{R} \mathrm{j} \mathrm{k} \mathrm{t} \mathrm{n} \delta \eta \nu$ comfieri $\mathrm{M}^{\mathrm{A}}$ confier $\mathrm{p}$ : quod fieri $\mathrm{M} \gamma^{1} \mathrm{~h}$ ? quo fieri $\mathrm{F}^{\mathrm{c}} \omega$ Le conferri o; 4.284 quae F M P p c g r t $\varepsilon$ v : et quae $\omega$ Le; 4.290 rebus sit $\mathrm{F} \mathrm{M} \mathrm{P} \mathrm{p} \mathrm{c} \mathrm{g} \mathrm{q} \mathrm{r} \mathrm{o} \delta \varepsilon \eta v$ : sit rebus $\omega$ Le; 4.682 ex(s)tinxti $\mathrm{F}$ P t (extinxiti $\mathrm{M}$, extincxti ut vid. $\mathrm{M}^{\mathrm{A}}$, extinxit ut vid. $\left.\mathrm{M}^{\mathrm{P}}\right)$ : extincti $\mathrm{p}$ extinxi $\omega \gamma$ Le; 5.184 mnestheique Heinsius : mnesthique $\mathrm{P} \gamma$ mnestique M P p g r : mnest(h)eoque $\omega \gamma^{1}$ Le; 5.247 optare MPR V pa i jkq r tw x y y n o $\delta \varepsilon v$ : aptare b c d ef g h v z $\gamma^{1} \eta$ Le; 5.398

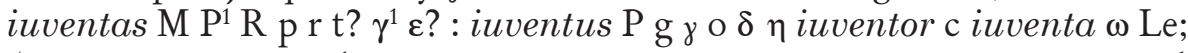
5.649 eunti M P R p b c i t o $\delta \varepsilon v$ : euntis $\omega$ Le; 6.209 crepitabat M P R d $\mathrm{w} \gamma^{1} \mathrm{n} v$ : crepitabant $\omega \gamma$ Le; 6.297 cocyto (vel cocito) M P R e j k t u v $\Lambda$ : cocyti (vel cociti) $\Phi \zeta$ Le; 6.438 tristique $\omega$ Le : tristisque $\mathrm{M}^{\mathrm{P}}$ (trisque $\left.\mathrm{M}\right)$ P R a b r $\gamma$ o $\varepsilon^{\text {ac }} \zeta v^{\mathrm{ac}} ; 7.173$ hic M P R a g r t $\Lambda$ : hinc $\omega \gamma^{1} \zeta$ Le; 7.447 deriguere $\mathrm{F} \mathrm{M} \gamma \mathrm{R}$ a j $\delta \zeta$ : diriguere $\omega \mathrm{Le} ; 7.773$ ad M P R b g j r $\gamma \zeta \eta v$ : in $\omega$ $\gamma^{1}$ Le; 8.70 sustinet M P R a r n o $\delta \eta v$ : sustulit $\omega \gamma^{1}$ Le; 8.672 spumabant $\omega$ Le : spumabat M P R b j r t x y $\gamma$ o $\zeta \eta v ; 8.724$ mulciber M P R i o n 
: mulcifer $\omega$ Le; 9.252 laudibus istis M P R b c e i j k v y $\gamma \varepsilon \zeta$ v: talibus ausis $\omega \gamma^{1}$ o Le; 9.456 spumantis M P R b d f g h r w z $\gamma \mathrm{n}$ o v spumantes $\delta \eta$ : spumanti $\omega \gamma^{1}$ Le; 9.634 traicit $\mathrm{M}$ b f $\zeta \eta \mathrm{v}^{\mathrm{pc}}:$ transigit $\mathrm{P} \mathrm{n} \delta$ transiit $\mathrm{P}^{\mathrm{X}} \gamma$ transit $\mathrm{n}^{\mathrm{ac}}$ transadigit $\mathrm{R}$ a transagit $\varepsilon$ traiecit $\omega \gamma^{1}$ Le; 10.6 quianam M P R V a b? c g j k v $\gamma$ n $\delta \varepsilon v:$ quaenam $\omega$ Le; 10.352 accurrit M P R a c $\mathrm{j}^{\mathrm{ac}} v$ : occurrit $\omega \mathrm{Le} ; 11.57$ ei M P b j k $\gamma \mathrm{n} \delta \varepsilon$ hei $v$ : et $\mathrm{R}$ a heu $\omega$ Le; 11.268 devictam asiam M P R a b o r y $\gamma \zeta$ : devicta asia $\omega \gamma^{1}$ Le; 11.281 portatis M P R a k r v n o $\delta \zeta:$ portastis $\omega$ Le; 11.654 derigit $\mathrm{M} \gamma \mathrm{R}$ a : degerit $\mathrm{b}$ adegerit d dirigit $\gamma^{1} \omega$ Le; 11.691 aversum M P R a f g k r x n o $\delta$ v:adversum $\omega$ Le; 11.839 multatam $\omega \gamma^{1} \delta$ Le : mulcatam M P R a? b d r $\gamma$ o vac punitam $\varepsilon ; 12.76$ refer $\mathrm{M} \gamma^{1} \mathrm{R}$ a c k o r u v y $\varepsilon$ : refert $\gamma \omega$ Le; 12.490 derigit M P R V $\mathrm{a}$ : dirigit $\omega \gamma \mathrm{Le} ; 12.801$ ne $\mathrm{M} \mathrm{P}^{2}$ a b i j o $\gamma:$ ni $\mathrm{P}$ nec $\mathrm{a}^{2} \omega$ Le.

Per una più accurata definizione dei rapporti tra il Leidensis e gli altri manoscritti carolingi ho estratto una selezione di varianti, che possono meglio rappresentare i legami di parentela o familiarità con i tre gruppi di Kaster. Mi è sembrato opportuno e più stringente evitare quelle varianti che sono conservate anche da uno o più manoscritti tardoantichi, come ad es.:

Aen. 2.90 pellacis M V c h k q t v $\gamma^{1} \Lambda$ : fallacis $\mathrm{P} \Pi_{5} \Phi \gamma \varepsilon$ Le; 2.349 audentem $\mathrm{P} \omega \gamma^{1}$ Le : audendi $\mathrm{M}$ b c? d? f h? i q r? $\gamma \zeta ; 4.490$ movet $\mathrm{M} \mathrm{P}$ o p t $\delta \varepsilon \eta$ Le : movit $\mathrm{F}$ ciet $\mathrm{F}^{\mathrm{c}} \mathrm{P}^{2} \omega \gamma ; 5.228$ fragoribus $\mathrm{M} \mathrm{R}$ p b c g j k r s x o $\delta \varepsilon \eta v$ : clamoribus $\Pi \omega \gamma$ Le; 6.265 tacentia F M P R a b c r $\gamma^{1}$ n o $\delta \eta$ Le : silentia $\mathrm{M}^{2} \mathrm{P}^{2} \omega \gamma^{2} ; 7.324$ dearum $\mathrm{F} \mathrm{M} \gamma$ a b? e f g r n o $\delta \zeta \eta$ Le : sororum $\mathrm{M}^{2} \gamma^{1} \mathrm{R} \omega ; 7.638$ trementis $\mathrm{F} \mathrm{M} \gamma \mathrm{R}$ a b e jac $\mathrm{r} \mathrm{u} n$ o $\zeta v$ Le trementes $\delta \eta$ : frementis $\mathrm{M}^{2} \gamma^{1} \omega ; 8.357$ arcem M P $\omega \gamma$ Le : urbem $\mathrm{M}^{\mathrm{A}} \mathrm{R}$ a d f i j r w z n o $\delta \varepsilon \eta v^{2 s l} ; 8.555$ litora M R a b d h i j r t w z $\gamma^{1}$ o $\delta \zeta \eta v$ Le : limina P $\omega \gamma$; 9.151 late $\mathrm{F} \mathrm{R}$ b j r Le : summae M P $\omega \gamma$ summe $\mathrm{F}^{\mathrm{c}} \delta \varepsilon ; 9.155$ ferant $\mathrm{F} \mathrm{P} \mathrm{c}$ ? o r x y $\gamma \eta$ : putent M R $\omega \gamma^{1}$ Le; 9.651 albos M P b c? f v x y $\gamma \mathrm{n} \mathrm{O}^{2} \varepsilon \zeta \eta \nu$ alvos $\delta$ : flavos $\mathrm{R} \omega \mathrm{Le} ; 10.18$ rerumque M P R V a r y $\gamma \eta$ Le : divumque $\omega$; 10.390 arvis M R $\omega \gamma^{1}$ Le arbis $\eta$ : agris $\mathrm{P} \mathrm{n}$ r x y z $\gamma \delta \varepsilon ; 11.202$ fulgentibus $\mathrm{R}$ a e o $\mathrm{u}$ : ardentibus M P $\omega \gamma$ Le; 12.178 coniunx $\mathrm{P} \mathrm{R}$ a b f g j o x $\gamma \zeta$ : iuno M $\omega \gamma^{1}$ Le.

In casi come questi è impossibile determinare se la variante di Le dipenda dalla familiarità con uno dei gruppi dei mss. carolingi oppure - più o meno direttamente - da uno degli antiquiores giunti fino a noi.

Per un motivo analogo non ho riportato quelle varianti di Le che sono sì attestate dalla sola tradizione carolingia ma da mss. di due o addirittura di tutti e tre i gruppi di Kaster, come: 
Buc. 5.45 nobis carmen e r Le (l'inversione, ora accolta da Ottaviano, è attestata da Probo 233.32 e piacque per l'eufonia ad Heyne con l'approvazione di Ribbeck $\left.{ }^{61}\right)$ : carmen nobis P R $\omega \gamma$.

Aen. 7.712 rosea $\mathrm{R} \Phi$ : rosa $\mathrm{M}$ roscia $\mathrm{P} \gamma$ roscida a w z $\gamma^{1} \Lambda$ Le; 8.527 increpat M P R $\omega$ : intonat a c e h i k u v y Le insonat $\mathrm{f} \mathrm{g} \mathrm{x;} 9.189$ soluti $\mathrm{M}$ P R $\omega$ : sepulti a d h i o s t w z $\varepsilon$ Le; 9.236 sepulti a e f v x $\varepsilon \zeta$ Le : soluti M $\mathrm{P} \omega \gamma$ solutis $\mathrm{R} ; 10.242$ ipse M P R V $\mathrm{V}^{2} \omega \gamma$ : igni $\mathrm{V}$ ingens $\mathrm{c} d \mathrm{e}$ h i t u v Le; 12.353 prospexit $\mathrm{M} \mathrm{P} \mathrm{R}$ a e $\mathrm{u} \mathrm{v}$ : conspexit $\omega$ Le.

Familiarità di Le col II Gruppo di Kaster ${ }^{62}$ :

Buc. 1.79 hic tamen hanc mecum poteras requiescere noctem poteras : poteris $\mathrm{d} \mathrm{n}^{2} \mathrm{Le}$

Questa lez., tipica dei manoscritti umanistici, è attestata anche in Arusiano e Pseudo Acrone.

Georg. 3.248-249 per silvas; tum saevus aper, tum pessima tigris; / heu male tum Libyae solis erratur in agris

Le con b g j r t $\Lambda$ si accorda con gli antiquiores M P R nel leggere per silvas, mentre $\Phi \zeta$ hanno per silvam; nel verso dopo, in clausola, Le segue la versione dei carolingi, che hanno in arvis (anche $\delta$ ), contro in agris di $\mathrm{M}$ $\mathrm{P} \mathrm{R}$ b r $\Lambda$. Se ne può dedurre una più stretta vicinanza di Le a g j $\mathrm{t}$ (codici del II gruppo).

Georg. 4.148 praetereo atque aliis post me memoranda relinquo

me memoranda $\mathrm{b}^{2} \mathrm{~h}^{2} \mathrm{i}^{2} \mathrm{j}^{2} \mathrm{w}^{2}$ : memoranda $\mathrm{M} \mathrm{P} \omega \gamma:$ commemoranda cd jktw z \& $\eta \mathrm{Le}$

Aen.3.581 et fessum quotiens mutet latus, intremere omnem mutet M P V a b f g z n $\eta$ o Tib. : mutat $\mathrm{M}^{\mathrm{A}} \mathrm{P}^{2}$ i $\mathrm{r} \gamma \varepsilon \zeta v$ agnoscit Serv. motet $\mathrm{cd}$ e v motat h j k x y $\gamma^{1} \delta$ Le Serv. nitet $\omega$

Aen. $\mathbf{5 . 7 5 6}$ sortiturque domos; hoc Ilium et haec loca Troiam troiam M P R m c h i k o r $\gamma$ Tib. : troia $\omega$ troiae b? t $\delta \varepsilon \eta \nu$ Le

Aen.9.632 effugit horrendum stridens adducta sagitta

adducta M R a b e f j k r u n $\delta \varepsilon \zeta \eta v$ : adlapsa $\mathrm{P} \gamma$ Tib. elapsa $\mathrm{cd} \mathrm{g} \mathrm{h}$ is t v w x y z Le

${ }^{61}$ Ottaviano cita in apparato Lachmann ad Prop. 1.6.25 «male vulgo carmen nobis, quasi in nobis et fessis sit expressa anthitesis»).

${ }^{62}$ Affini al II Gruppo di Kaster possono considerarsi anche g k i w x y z. 
Aen. 10.86 est Paphus Idaliumque tibi, sunt alta Cythera paphus $\mathrm{M} \mathrm{P} \omega$ : paphys $\mathrm{R}$ paphos $\mathrm{d} \mathrm{f} \mathrm{h} \mathrm{k} \mathrm{o^{2 } \mathrm { t }} \mathrm{w} \mathrm{Le}$

Aen. 10.113 fata viam invenient. Stygii per flumina fratris invenient M P R $\omega$ : inveniant $\mathrm{c} \mathrm{d} \mathrm{g}$ h i $\mathrm{o}^{2} \mathrm{t} \mathrm{w} \gamma \mathrm{Le}$

Aen.10.602 talia per campos edebat funera ductor ductor M P R $\omega$ : doctor a $\gamma$ victor $\mathrm{cd} \mathrm{h} \mathrm{i} \mathrm{k} \mathrm{t} \mathrm{y} \gamma^{1} \eta \mathrm{Le}$

Aen. 10.673 quosne (nefas) omnis infanda in morte reliqui in morte M P R $\omega$ : morte d h i j o ${ }^{2}$ t Le Tib.

Aen. 10.686 continuit iuvenemque animi miserata repressit animi M P R $\omega$ : animo d t Le

Aen. 10.737 pars belli haud temnenda, viri, iacet altus Orodes viri M P R V $\omega$ : viris c d g t n $\delta \varepsilon \zeta L^{\text {ac }}$ Asper ap. Serv.

Al II Gruppo di Kaster avvicina Le anche l'inversione di Aen. 4.258-257 (come cdhijk tw x z).

Familiarità di Le con i mss. del I Gruppo di Kaster:

Georg. 1.197 vidi lecta diu et multo spectata labore spectata A M P R $\omega$ : expectata b Le

Aen. 4.528 lenibant curas et corda oblita laborum hab. $\mathrm{b} \mathrm{o}^{2} \gamma^{1} \varepsilon^{2 \mathrm{mg}} \mathrm{Le}^{63}$ Il verso che manca nei codici tardoantichi e in tutti gli altri medievali, è quasi identico a 9.225, con la variante lenibant al posto di laxabant, ed è considerato spurio da quasi tutti gli editori, ma ha trovato anche difensori illustri, cfr. l'apparato critico della Coll. Alma Mater ad loc.

Aen. 5.786 urbem odiis satis est nec poenam traxe per omnem omnem F M R $\omega$ : omnis $\mathrm{P}$ (corr. $\left.\mathrm{P}^{1}\right)$ omnes b? $\gamma \mathrm{Le}$

Aen. 6.509 ad quae Priamides: nihil o tibi, amice, relictum ad quae $\mathrm{F} \mathrm{R}$ at quae $\mathrm{M}$ (atque $\mathrm{M}^{\mathrm{X}}$, hic add. alio ut vid. atramento $\mathrm{M}^{2}$ ) $\mathrm{P}$ : atque b $\mathrm{r}$ Le Tib. atque hic a $\gamma$ atque (ad quae) haec $\omega \gamma^{1}$ ad quem $\mathrm{n}$

Aen. 8.244 infernas reseret sedes et regna recludat et M P R $\omega: a c$ b Le

${ }^{63} \mathrm{Il}$ verso è di solito attestato nei testimoni umanistici, cf. Venier, Per una storia, 7. 
Aen. 8.566 ter leto sternendus erat; cui tunc tamen omnis tunc M P R $\omega$ : tum b r Le nunc $\eta$

Aen. 12.335 Thraca pedum circumque atrae Formidinis ora ora M P R $\omega$ : oras b Le horas c

Familiarità di Le con i mss. del III Gruppo di Kaster:

Georg. 2.462 mane salutantum totis vomit aedibus undam vomit M P R $\omega$ : vomat $\mathrm{a}^{\text {ac }} \mathrm{Le}$

Aen. 8.397 tum quoque fas nobis Teucros armare fuisset tum M P R $\omega$ : tunc a e r u v Le

Aen. 8.421 stricturae Chalybum et fornacibus ignis anhelat et M P R $\omega$ : om. a Le

Aen. 8.435 aegidaque horriferam, turbatae Palladis arma horriferam M P R $\omega$ : horrificam a? $\gamma^{1}$ Le

Aen. 8.559 haeret, inexpletus lacrimans, ac talia fartur

inexpletus $\mathrm{M} \mathrm{P}^{(1)} \mathrm{b} \mathrm{d} \mathrm{j} \mathrm{r} \mathrm{t} \mathrm{w} \mathrm{z} \gamma \eta$ : inexpletum $\omega \gamma^{1}$ inpletus $\mathrm{R}$ in ample$x u$ a c? $\zeta$ Le in amplexum $\mathrm{n}^{2 \mathrm{sl}}$

In Le si legge in amplexu, con compendio sulla $u$, che potrebbe essere stato aggiunto successivamente.

Aen. 9.72 atque manum pinu flagranti fervidus implet manum pinu $\mathrm{M} \mathrm{R} \omega$ : manu pinum $\mathrm{P} \gamma$ manum pinum a Le

Aen. 9.290 at tu, oro, solare inopem et succurre relictae at M P R $\omega$ : hanc a c u $v \varepsilon \vee$ Le

Aen. 11.637 hastam intorsit equo ferrumque sub aure reliquit reliquit $\mathrm{M} P \mathrm{R} \omega$ : relinquit a e $\mathrm{r} \mathrm{u} \mathrm{v}$ Le

Aen. 11.759 Maeonidae incurrunt. Tum fatis debitus Arruns tum fatis $\mathrm{M} \gamma \omega$ : fatis tum a e $\mathrm{v}$ Le fatis tantum $\mathrm{u}$

Cfr. anche Georg. 4.449 lassis M P V $\omega \gamma$ : lapsis R a o (ex lapsus) $\varepsilon$ Le; Aen. 5.162 cursum M ${ }^{\mathrm{A}}$ ? $\omega$ : gressum M P R a e v z $\gamma \eta$ Le; 9.645 mittit M $\omega \gamma^{1}$ : mittit et $\mathrm{R}$ a e j u Le misit $\mathrm{P}$ c v x y $\gamma ; 10.106$ licitum M P $\omega \gamma$ : licitum est $\mathrm{R}$ a c e g j u v $\delta \eta \mathrm{Le} ; 11.220$ ingravat $\mathrm{M} \mathrm{P} \omega$ : ingruat $\mathrm{R}$ a Le; 11.834 incurrunt M P $\omega$ : concurrunt $\mathrm{R}$ a e j ${ }^{\mathrm{ac}}$ Le; 11.882 inter $\mathrm{F}$ P $\omega$ $\gamma$ : intra M R a 
e u v z Le; 11.911 flatusque M P $\omega$ : flatumque $\mathrm{R}$ a e v Le; 12.16 ferro crimen $\mathrm{M} \mathrm{P} \omega$ : crimen ferro $\mathrm{R}$ a c e u v Le; 12.596 incessi $\mathrm{M}^{\mathrm{A}} \mathrm{P} \mathrm{d}$ i j k v x n $\delta \varepsilon$ : incedi $\mathrm{M}$ incensi $\mathrm{R}$ a Le ${ }^{\mathrm{ac}}$ incendi $\omega \gamma \mathrm{Le}^{\mathrm{pc}}$;

Caratterizzano Le ancora delle omissioni, delle aggiunte e delle inversioni di versi:

Omissioni:

Buc. $4.58(=\text { c })^{64} ; 8.28$ a (tutti i codd., eccetto $\left.\gamma\right)$

Aen. 1.81-84 [solo Le, una seconda mano supplisce nel margine inferiore]; Aen. 3.171-172 ausonias attonitus visis et voce deorum (Le presenta una conflazione di 171 - solo ausonias - e 172 - attonitus ... deorum); Aen. 3.204a-204b-204c (tutti i codd.); Aen. 4.436 (soltanto Le); Aen. 5.790 (soltanto Le); Aen. 9.29 (tutti i codd.); Aen. 9.529 (come quasi tutti i codd.: lo leggono soltanto $\mathrm{R} \mathrm{a}^{2} \mathrm{j} \mathrm{o}^{2}$ ); Aen. 10.278 (=9.127; Le omette il verso come $\mathrm{M}$ $\mathrm{Pdf} \mathrm{g} h \mathrm{ik} \mathrm{t} \gamma \mathrm{n} \varepsilon \zeta \mathrm{v}$; lo aggiunge nell'interlineo, forse la stessa mano; il verso si legge in $\mathrm{R} \mathrm{b} \mathrm{c} \mathrm{e} \mathrm{j} \mathrm{o} \mathrm{o}^{2} \mathrm{u}$ v y z $\left.\gamma^{1} \delta \eta\right)$; Aen. 10.872 (=12.668, Le omette il verso come M P R a b n r $\gamma$; lo aggiunge un'altra mano; il verso si legge in c d e f g h i j k o t u v x y z $\gamma^{1} \delta$ (dopo il v. 873) $\zeta v$ ).

Aggiunte:

Georg. 1.389a (Le ha il verso come $\mathrm{M}^{\mathrm{P}}$ (ima pagina) $\gamma^{1}$ (in marg.), ma prima del verso $389^{65}$, quindi: aut caput obiectat querulum venientibus undis [aut Le $\gamma^{1}$ : et $\left.\mathrm{M}^{\mathrm{P}}\right]$ / et sola in sicca secum spatiatur harena; Georg. 4.338 (Le ha il verso come a c d w o $\left.\delta \eta \gamma^{1}\right)^{66}$; thaliaque: thalique a $\mathrm{c} \mathrm{d}$ w et talique $\gamma^{1}$ Le; Aen. 4.273 (Le ha il verso come c de j k r t v w x y z $\gamma^{1} \delta \varepsilon^{2 \mathrm{mg}}$ $\eta$; il verso è aggiunto anche da $\left.\mathrm{M}^{\mathrm{A}}\right) ;$ Aen. 4.528 (= 9.225 con lenibant al posto di laxabant; Le ha il verso come $\mathrm{b} \gamma^{1} ; \mathrm{M}^{\mathrm{A}}$ lo aggiunge e poi lo cancella, cfr. supra); Aen. 6.242 (Le legge il verso come $\mathrm{R} \mathrm{b} \mathrm{k} \mathrm{x} \mathrm{o} \delta^{2 \mathrm{mg}} \zeta^{2 \mathrm{mg}} \eta \nu$, prima del v. 241 a $\gamma$, cfr. supra); Aen. 7.760 lacus : et Megarus ipse (questa aggiunta solo in Le, cfr. supra); Aen. 8.46 (Le ha il verso come R b c d e f g h i j k s t v w x y z $\gamma^{1}$ o $\left.\delta \varepsilon \zeta \eta\right)$; Aen. 12.612-613 (=11.471-472, ha i versi come c d ef gi j k o $\mathrm{O}^{2} \mathrm{vxyz} \varepsilon$ ).

\section{Inversioni:}

Georg. 2.345-344 (soltanto Le); Georg. 4.291-292-293 (come quasi tutti i codd. medievali); Aen. 3.526-525 (soltanto Le); Aen. 4.258-257 (come c d h i j k t w x z); Aen. 5.777-778 (come quasi tutti i codici medievali).

${ }^{64} \mathrm{Il}$ cod. c omette Buc. 4.58, che una seconda mano supplisce nel margine destro.

${ }^{65}$ Così anche $\mathrm{f}^{1} \mathrm{e}$ i correttori più recenti di $\mathrm{g}$ i k s z.

${ }^{66} \mathrm{Il}$ verso è ampiamente accolto nei manoscritti umanistici, cf. Venier, Per una storia, 6. 
Concludiamo il nostro esame del ms. Leidensis stabilendo se esistono rapporti più stretti di Le con $\mathrm{n}$ e gli altri codici in beneventana, usando gli apparati critici allestiti da Ottaviano e Conte per l'ed. delle Bucoliche e delle Georgiche e, come abbiamo già fatto supra, nostre collazioni (di o $\vee \delta \varepsilon \zeta$ ๆ) per l'Eneide.

Buc. 1.12 turbatur $\mathrm{k}$ y z $\Lambda \mathrm{Le}^{67} ; 1.13$ protenus $\mathrm{b} \mathrm{d} \mathrm{k} \mathrm{r} \mathrm{x} \mathrm{n} \gamma$ Le; 2.57 concedet $\mathrm{d} \mathrm{k} \mathrm{r}$ v $\Lambda$ Le; 3.26 iuncta $\varepsilon$ Le : vincta $\mathrm{P} \mathrm{R} \omega \gamma$ : Mynors accoglieva iuncta sulla scorta di $\mathrm{P}$, ma Geymonat a ragione precisa che il Palatinus legge vincta e così tutti i mss.; iuncta - preferita da molti editori precedenti - è invece in due passi di Rufino, 42.3 e 61.30 e nel lemma di Serv. Buc. 3.2568; 3.38 facili i k z $\Lambda \gamma^{1}$ Le; 3.51 lacesses $\Lambda$ Le;

Georg. 1.320 sublime b c d e i 1 z $\Lambda$ Le; 1.354 quod a c d $\Lambda \gamma^{1}$ Le; 2.131 arbor a c e ikrst v $\Lambda$ (praeter $\eta$ ) $\gamma^{*} \gamma^{2}$ Le; 2.247 amaror $\mathrm{M}^{2}$ b k t v y $\Lambda \mathrm{Le}^{69}$; 2.281 directaeque a i k s t z $\Lambda$ (praeter o) $\gamma^{1}$ Le; 2.330 tepentibus M t $\Lambda$ Le; 2.359 bicornes $\mathrm{V}$ d e i j s t x z $\Lambda$ Le; 2.425 placidam a d z n $\delta \eta$ Le; 2.479 tumescunt $\mathrm{n} \mathrm{Le}{ }^{70} ; 3.63$ iuventus $\mathrm{Mg}$ h $\Lambda$ Le; 3.69 mavis $\mathrm{M} n$ oc $\delta$ Le; 3.194 provocet $\mathrm{P}$ t $\Lambda$ Le; 3.415 gravi $\mathrm{k} n$ o Le; 3.435 nec $\mathrm{M}$ a $\mathrm{n} \varepsilon$ Le; 4.43 fodere $\mathrm{M}^{\mathrm{x}} \mathrm{t} \Lambda \mathrm{Le} ; 4.190$ suus sopor $\mathrm{n} \mathrm{Le}{ }^{71} ; 4.281$ quem M P R b j r t $\Lambda$ (praeter o ${ }^{\text {ac }}$ ) Le; 4.370 saxosumque c i $\mathrm{k} \mathrm{r}$ ? t v w x y z $\Lambda$ Le; 4.439 vinclisque a e $\mathrm{j}$ v $\mathrm{n}$ $\delta \gamma^{1} \mathrm{Le}$

Aen. 2.554 haec: hic in $\delta \zeta$ Le; 2.755 animo M P a $\chi$ n o $\delta \eta \nu$ Le; 3.478 praeterlabere $\mathrm{M}$ a i $\mathrm{n}$ o $\delta \zeta v^{\text {ac }}$ Le; 3.483 subtegmine $\mathrm{G} \mathrm{M} \mathrm{g?} \gamma$ n o $\delta \varepsilon \eta v$ Le; 3.516 pliadasque d $\mathrm{w} \gamma^{1} \mathrm{n}$ o $\varepsilon \zeta \eta$ Le; 3.545 aram $\mathrm{P}$ r $\gamma$ n o $\delta \eta \nu$ Le; 4.18 fuisset $\mathrm{F} \mathrm{P}^{\mathrm{x}} \mathrm{R} \mathrm{p}$ i $\gamma^{1} \mathrm{n} \mathrm{o}^{\mathrm{pc}} \delta \zeta \eta v$ Le; 6.602 cadenti $\mathrm{R} \Pi_{10} \gamma \mathrm{n} \delta \eta$ Le; 9.371 muroque M R g j n $\delta \varepsilon \zeta v^{\mathrm{pc}}$ Le; 10.675 dehiscit $\mathrm{n} \delta \varepsilon$ Le.

Alla luce di quanto si è sinora cercato di esporre, il ms. Leidensis (Le) rappresenta senz'altro un interessante testimone virgiliano d'età carolingia, ulteriore tassello della difficile ricostruzione della tradizione manoscritta virgiliana medievale, che potrebbe forse trovare posto nell'apparato critico di una futura edizione di Virgilio, se tali apparati tenderanno sempre più a fornire un'ampia messe di varianti. H.

${ }^{67}$ Questa variante è tipica dei manoscritti umanistici, cf. Venier, Per una storia, 6.

${ }^{68}$ Per Servio si rimane incerti, perché già l'incompleto apparato di Thilo segnala vincta in

${ }^{69}$ La variante amaror, preferita nei codici umanistici (cf. Venier, Per una storia, 6) ha fatto molto discutere studiosi ed editori di Virgilio, cf. S. Timpanaro, Virgilianisti antichi e tradizione indiretta, Firenze 2001, 17-21.

${ }^{70}$ Non ho potuto ricontrollare il cod. $\mathrm{n}$. Geymonat dice che $\mathrm{n}$ legge tumescunt; Conte in apparato non ha nulla, bisognerebbe quindi dedurre che il consensus codicum $-\mathrm{n}$ incluso - ha tumescant.

${ }^{71}$ L'inversione in n è registrata dal solo Geymonat, cf. la nota 70. 